\title{
Liver clot after flap surgery: A case report
}

\author{
Jayakrishnan. R', Aneesh Alim ${ }^{2, *}$, Basim Burhan K.B ${ }^{3}$ \\ ${ }^{1}$ Private Practitioner, Dept. of Oral \& Maxillofacial Surgeon ${ }^{2}$ Associate Professor, Dept. of Orthodontics and Dentofacial \\ Orthopedics, PMS College of Dental Science \& Research Thiruvananthapuram, Kerala, ${ }^{3}$ Senior House Surgeon PMS College of \\ Dental Science \& Research, Thiruvananthapuram, Kerala, India
}

*Corresponding Author:

Email: drvishnu731@gmail.com

\begin{abstract}
The dentist may encounter different types of complication during the surgical procedures either intraoperative or post operatively. Of these complications, the most common is postoperative hemorrhage. The hemorrhage can be classified into primary, intermediate and secondary hemorrhage. Normally after periodontal flap surgery, the bleeding will stop post operatively which is stopped by primary wound closure. This case discusses a rare complication after periodontal flap surgical procedure called as "currant jelly" or "liver clot" and its management.
\end{abstract}

Keywords: Haemorrhage, Liver clot, Flap surgery complications.

\section{Introduction}

Flap surgery is one of the means which results in periodontal pocket depth reduction in cases with chronic and aggressive periodontitis and also considered as a common component of dental and periodontal specialty practice. After flap surgery different types of complication can be encountered by a periodontist such as post-operative hemorrhage, pain, recession, hypersensitivity etc. significant hemorrhage after periodontal flap surgical procedure is not common unless the patient who underwent surgical procedure is having any systemic disorder associated with any clotting mechanism. ${ }^{1}$

Post-operative surgical complication associated with periodontal procedures can be grouped into two different categories such as: those complications related to bleeding and those complication related to delayed wound healing and infection. ${ }^{2}$ A periodontal surgical procedure put forward different challenges to the body's clotting mechanism.

The reason for considering the surgical procedures as challenges to the hemostatic mechanism is because of the following:- first intraoral tissue are highly vascular, second the patient's tongue tends to dislodge the clot that is formed after periodontal surgical procedures which results in initiation of secondary bleeding. The other factors that have to be considered is the role of salivary enzymes that will leads to the breakdown of blood clot which ultimately will prevent the clot organization as well as ingrowth of granulation tissue. $^{3}$

The following report addresses a rare case of "liver clot" or "currant jelly clot" after periodontal soft tissue flap surgery

\section{Case Report}

A 32 year old female patient reported to the dental clinic with the chief complaint of redness and swollen gums. The patient also reported that he had bleeding from the gums while brushing and chewing on hard food stuffs. Periodontal evaluation revealed the final diagnosis of chronic periodontitis. The patient is systemically healthy and there is no history any past noteworthy illness such as disorder associated with blood coagulation, liver disorder, prolonged intake of medication or hospitalization.

Surgical Procedure: After routine blood examination, the patient underwent periodontal flap surgery in her first quadrant teeth and bone replacement graft in between second premolar and first molar teeth 3 weeks after non-surgical periodontal therapy. Post-surgical medications and homecare instructions were given to the patient.

Post-operative Quiddity: The following morning after flap surgery, the patient again reported with a massive clot in her posterior tooth. Upon clinical examination, dark red jelly like pedunculated mass which is firmly attached to the upper right first molar and canine teeth was noticed (Fig. 1). The mass was removed using a 9/10 Graceycurette (Hu friedy) (Fig. 2). It was irrigated with a mixture of betadine and saline and finally pressure pack was applied. No further bleeding was evident after the application of pressure pack. The patient had no further postsurgical complications.

The excised mass was clinically determined as "liver clot" or "currant jelly clot".

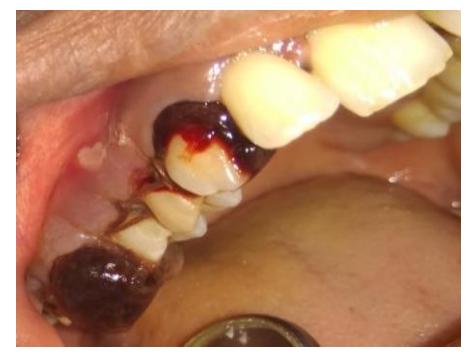

Fig. 1: Liverclot in the upper right canine and first molar 


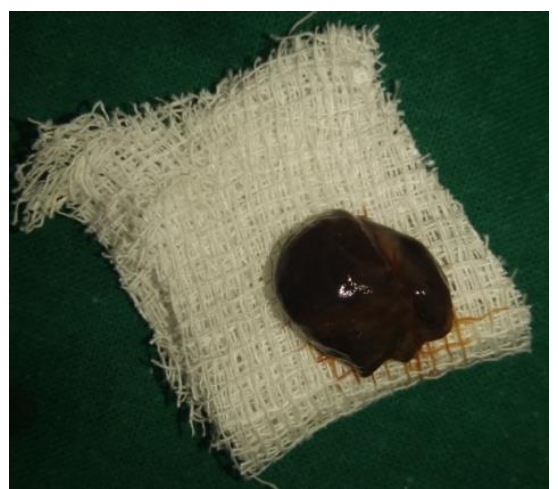

Fig. 2: Excised liver clot

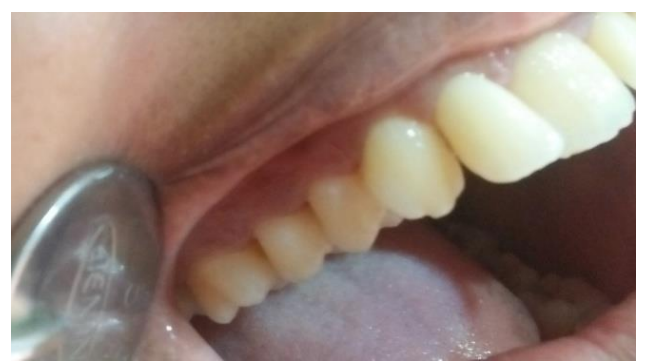

Fig. 2: 1 week postoperative view

\section{Discussion}

Hemorrhage, a life threatening complication that occurs intraoperatively or postoperatively after minor and major oral surgical procedures. Hemorrhage is an uncommon postoperative complication after periodontal flap surgical procedures. ${ }^{4}$

Depending on the occurrence, the hemorrhage can be classified as primary, intermediate and secondary hemorrhage. Primary hemorrhage occurs intraoperatively as a result of rupture or wounding of the blood vessels. The hemorrhage that occurs within 24 hours as a result of removal of pressure pack or due to vasoconstrictive agent dissipation is called as intermediate hemorrhage. The hemorrhage that occurs after 24 hours due to infection trauma, foreign bodies, portion of dental restorative material or bone replacement graft that will interfere with clot organization is called secondary hemorrhage. ${ }^{5,6}$

Normally after periodontal flap surgery, the process of blood coagulation and fibrinolysis takes place. Immediately after vessel injury, there will be adherence of platelets in the subendothelial tissue at the injury site. They aggregate to form the primary hemostatic plug. These platelet cause the activation of clotting factors leading to the formation of fibrin clot that reinforces platelet aggregate. ${ }^{7}$ Hence platelet are key players in hemostasis. The insufficient number of platelets results in inability of blood to form clots. This can lead to oozing of blood from periodontal wounds. ${ }^{8}$

The excised mass was clinically determined as "liver clot" or "currant jelly clot" and for confirmation a biopsy was done. The biopsy reported the presence of fibrous band surrounded by erythrocytes.
Liver clot which is characterized by slow, oozing dark hemorrhage and is rich in hemoglobin from erythrocytes which is rich in red clot.

In the present case report, the reason for the formation of liver clot I may be due to bone graft palced in the region or may be due to continuous irritation of the surgical site by the patients tongue as the patient is aware that there some medicine has been placed. That irritation disrupted the normal clotting mechanism which ultimately resulted in formation of liver clot.

\section{Management}

Formation of liver clot is a rare instant. However the dental specialist should be proficient in and also should have thorough knowledge about the etiology and management. In the present case we did a normal curettage of the area of the interest followed by irrigation with betadine and saline and isolated with periodontal pack. After 1 week the patient reported to the clinic without no evidence of any sequale and there is proper healing started to take place (Fig. 3 )

However, in emergency situation like if the patient is having pain we can use soft tissue laser not only for removing the blood clot but also to enhance healing utilizing low level laser therapy. ${ }^{9-13}$

\section{Conclusion}

There are dental treatment procedures having potential of causing life threatening complications. The dentist should take consideration regarding systemic health of patient, the dentist should take care of surgical anatomy and the most important is dentist should inform the patient about the possible chances of postoperative complication to occur. In case of liver clot other than conventional treatment protocol such as pressure, ice pack, different hemostatic agent etc. Laser application seems to be very beneficial.

\section{References}

1. Richard F. Post-surgical hemorrhage: Formation of a "liver clot" secondary to periodontal plastic surgery. $J$ Contemp Dent Pract 2001;2;1-6

2. Pandya D, Manohar B, Mathur LK, Shankarapillai R. "Liver clot"-A rare periodontal postsurgical complication. Indian J Dent Res 2012;23:419-22.

3. Pertson L. J. (editor). Contemporary Oral and Maxillofacial Surgery, 3rd edition. C.V. Mosby Company, St. Louis. Chapter 3 "Principles of surgery"; Hupp JR.1998;48-49. Chapter 11 "Prevention and management of surgical complications"; Peterson LJ.1998;270-273.

4. Kruger GO, editor. Hemorrhage and shock"; Alling CC III, Alling RD. Textbook of Oral and Maxillofacial Surgery. 6th ed., Vol. 229, Ch. 12. St. Louis: The C.V. Mosby Company; 1984. p. 236-9.

5. Lapeyrolerie F. Management of dentoalveolar hemorrhage. Dent Clin North Am 1973;17:523-32.

6. Pandya D, Manohar B, Mathur LK, Shankarapillai R. "Liver clot": A rare periodontal postsurgical complication. Indian J Dent Res 2012;23:419-22. 
7. Post-Surgical Hemorrhage: Formation of a "Liver Clot" Secondaryto Periodontal Plastic Surgery. The Journal of Contemporary Dental Practice 2(2).

8. Natalia E, Irene B. Comprehensive Approach in "Liver Clot" Management Case Report. Biomed J Sci \& Tech Res 3(5)-2018.

9. Andrew J Gale (2011) Current Understanding of Hemostasis. Toxicol Pathol PMC 39(1):273-280.

10. Atanaska Dinkova, Donka Kirova, Delyan Delev (2013) Dentalmanagement and bleeding complications of patients on long-termoralantiplatelet therapy. Review of existing studies and guidelines. $J$ of IMAB19(2):298-304.
11. Hassan G Moghadam, Marco F Caminiti (2002) LifeThreatening Hemorrhage after Extraction of Third Molars: Case Report and Management Protocol. J Can Dent Assoc 68(11):670-674.

12. James M Henderson, Stewart Bergman, Andrew Salama, Gary Koterwas(2001) Management of the Oral and Maxillofacial, Surgery Patient with Thrombocytopenia. J Oral Maxillofac Surg 59(4):421427.

13. Richard F Duckman, Edward B Fowler, Lawrence G Breault (2001) Post Surgical hemorrage formation of a Liver Clot Secondary to periodontal Plastic Surgery. The Journal of Contemporary Dental Practice 2(2). 